\title{
LES CONNECTEURS EN COMBINAISON AVEC LES MARQUEURS MODAUX: L'EXEMPLE DU FRANÇAIS ET DU SLOVÈNE'
}

\section{Introduction}

En analyse discursive, les unités syntaxiques traditionnelles, comme la phrase, aussi bien que les unités pragmalinguistiques, comme l'acte de parole, ne peuvent pas répondre à la question: "Quelles sont les unités reliées par les connecteurs discursifs?"

Il existe plusieurs théories à la base cognitive dans lesquelles les auteurs essaient de définir les unités discursives minimales, comme par exemple D. Schiffrin (1987) avec l'unité discursive, A. Berendonner (1990) avec la théorie de la mémoire discursive ou p. ex. J. M. Luscher avec l'approche de la pertinence, aussi bien que C. Rossari qui combine les théories de Schiffrin et de Berendonner. Toutes ces théories sont problématiques, parce que aucune d'elles n'arrive à établir une unité stable et généralement applicable: aucune d'elles ne répond entièrement au problème de l'unité d'analyse (Schlamberger Brezar, 1998). J. Moeschler (1998: 77), dans sa définition des connecteurs, dit même que:

"un connecteur pragmatique est une marque linguistique, appartenant à des catégories grammaticales variées (conjonctions de coordination, conjonctions de subordination, adverbes, locutions adverbiales), qui articule des unités linguistiques maximales ou des unités discursives quelconques..."

En même temps, il existe une approche linguistique globale de M. A. Morel (Morel, Danon-Boileau, 1998) où est introduite une unité d'analyse, qui est le fruit d'une discipline pluridisciplinaire, associant phonétique et prosodie, morphosyntaxe, énonciation et analyse de discours. C'est l'unité du discours oral spontané, l'unité maximale où l'on peut toujours parler d'une "grammaire". ${ }^{2}$ Les limites du paragraphe sont données par l'intonation. Chaque paragraphe se compose à son tour d'un ou de plusieurs constituants discursifs: d'un rhème, qui est, régulièrement, précédé d'un ou plusieurs préambules. Le dernier rhème peut être suivi d'un postrhème.

Ce qui nous intéresse le plus, c'est la place des connecteurs dans le paragraphe oral. Les connecteurs, nommés les ligateurs dans la théorie de $\mathrm{M}$. A. Morel, servent à baliser l'oral et relient ce qui va suivre avec ce qui a été dit précédemment. Ils sont situés

1 Cette communication est la version complétée de la conférence, donnée en anglais au mois de juillet 1999 à Ljubljana au cours du 32ième congrès de la Societas Linguistica Europea.

2 La grammaire est mise entre guillemets parce qu'il ne s'agit pas d'une grammaire au sens traditionnel du terme mais plutôt d'une structuration canonique. 
au début du paragraphe et se combinent très souvent avec les adverbes de modalité épistémique ou appréciative, comme par exemple alors effectivement ou mais justement.

La recherche qui a été menée pour être présentée dans cet article, est basée sur deux hypothèses:

- premièrement, le paragraphe oral, en tant qu'unité du discours spontané où les marqueurs de modalité et connecteurs jouent un rôle essentiel, peut être défini dans $d^{\prime}$ 'autres langues ${ }^{3}$ et aussi en slovène.

- deuxièmement, en français et en slovène, les marqueurs modaux qui se combinent avec les connecteurs aparaissent en combinaisons régulières et ont dans la langue une fonction qui leur est propre.

La recherche est basée sur l'analyse du corpus, formé de 6 heures de discours oral authentique en langue française, notamment des débats télévisés (Polémiques, Bouillon de culture), et 5 heures de simulations de négociations en langue slovène. Tous les exemples cités dans l'article, qui servent à démontrer les phénomènes de linguistique comparée, appartiennent à ces deux sources.

\section{Le paragraphe oral}

Dans sa Grammaire de l'intonation (Morel, Danon-Boileau, 1998: 21), M. A. Morel décrit le paragraphe oral de la manière suivante:

"Nous appelons paragraphe oral ${ }^{4}$ l'unité d'analyse de la parole spontanée, l'unité maximale susceptible d'une 'grammaire', au delà de laquelle les relations entre éléments relèvent de l'analyse de discours. Le choix du 'paragraphe' comme unité d'analyse de l'oral se justifie par l'analogie avec la définition qu'on en donne à l'écrit. De même qu'à l'écrit le paragraphe ne peut être défini que par des indices typographiques - alinéa au début et blanc à la fin (donc extérieurs au plan segmental) - de même à l'oral seuls les indices suprasegmentaux permettent le découpage en paragraphes. ${ }^{5}$

\subsection{Le paragraphe oral français}

Chaque paragraphe se compose d'un ou de plusieurs constituants dans l'ordre suivant: le préambule, le rhème et le postrhème. Un paragraphe comprend au moins un rhème. Ce rhème est en français régulièrement précédé d'un ou de plusieurs préambules, qui sont des segments à valeur thématique et modale. Le dernier rhème du paragraphe peut être suivi d'un postrhème (exemple (1)):

Paragraphe oral type $=$ préambule + rhème + postrhème

3 Dans un article récent de M. A. Morel et ses étudiants (1997), il s'agit de la définition du paragraphe en japonais, thaï, anglais, turc et persan.

4 C'est l'auteur qui place le syntagme en italiques

5 C'est l'auteur qui souligne. 
(1)

mais c'est bon, lelle est décapotable,/ la bagnole.

(Morel, Danon-Boileau, 1998: 22)

La segmentation du paragraphe en constituants est indiquée par les indices segmentaux et suprasegmentaux. Les indices suprasegmentaux permettent seuls l'identification des paragraphes: la chute de l'intensité et de l'intonation représente la fin du paragraphe. ${ }^{6} \mathrm{La}$ remontée de l'intonation sur la syllabe finale d'un constituant ou d'un ensemble de constituants a pour effet rétroactif d'unifier cet ensemble, en lui conférant le statut de préambule du texte oral qui le suit, et cela quel que soit son statut discursif au plan segmental (Morel, Danon-Boileau, 1998: 23).

Les indices segmentaux permettent à leur tour de déterminer la nature des constituants discursifs à l'intérieur du paragraphe.

D'après M. A. Morel (Morel, Danon-Boileau, 1998: 21), le préambule en français est extrêmement décondensé, formé de plusieurs segments juxtaposés, dont chacun correspond à une fonction énonciative et discursive bien définie. Cette décondensation du préambule contribue à réduire l'extension du rhème, qui est le plus souvent très court en français (Morel, Danon-Boileau, ibid.).

Le préambule français se compose des éléments suivants: le ligateur, les indices de modalité qui peuvent être représentés par le point de vue ou modus dissocié à valeur épistémique ou appréciative, suit le cadre qui correspond au thème ou sujet et le support lexical disjoint (Morel, Danon-Boileau, 1998: 37). Ces éléments sont représentés dans l'exemple (2), cité par M. A. Morel et L. Danon-Boileau (1998: 39):

(2)

\section{Préambule}

- connecteur/ligateur

bon e je sais pas

- point de vue on décide

- modus dissocié qu'on va monter

- cadre un centre: un centre de formation français des professions

- support lexical disjoint alors e y a la prof

\section{Rhème}

qui va: qui va décider e si e bon qui organise au sein d'l'association des profs de français.

Pour un locuteur français, l'essentiel n'est pas tant d'insister sur ce qu'il dit de nouveau que de bien montrer le point d'où il part et la valeur qu'il accorde à ce point. Le

6 Pour le découpage en paragraphes, l'auteur se sert du logiciel ANAPROZ, construit à des fins de l'analyse suprasegmentale. Nous n'allons entrer en détail sur les spécifications phonétiques qu'en ce qui concerne les connecteurs et les marqueurs modaux. 
connecteur (ou le ligateur) précise le lien de ce qui va se dire avec ce qui a déjà été dit et signale en même temps quelle position va prendre l'énonciateur par rapport au coénonciateur d'après le schéma de la coénonciation. ${ }^{7}$ Dans le point de vue, l'identité de l'énonciateur, qui sert de garant de ce qui va être dit, est soulignée. Le modus dissocié apporte des précisions sur le degré de certitude de l'information que l'énonciateur va délivrer. Le modus dissocié peut avoir la valeur épistémique, comme p. ex. les adver-bes effectivement ou peut-être, ou bien les verbes avec lesquels l'énonciateur peut indiquer le degré de la vérité de ce qui vient d'être énoncé, comme p. ex. je crois, j'ai l'impression, il me semble. Le cadre définit le domaine dans lequel va se dérouler le propos. Et le support lexical disjoint met parfois en place la référence de l'argument qui sert de support à la prédication du rhème. Habituellement, ce support se trouve dissocié syntaxiquement et intonativement du rhème.

Nous pouvons conclure que, pour l'étude des connecteurs en combinaison avec des marqueurs de modalité épistémique dans le cadre du modèle de $\mathrm{M}$. A. Morel, le segment nommé le préambule est le plus important. Il arrive rarement en effet que tous les éléments du préambule soient présents dans un seul paragraphe. Il peut y en avoir un ou deux seulement.

\subsection{Le paragraphe slovène}

L'unité du paragraphe est aussi applicable à l'étude de la langue slovène. Dans l'exemple (2), nous analysons en termes de paragraphe un segment intonatif, pris au discours oral:

No, mislim, da bi nam to ustrezalo.

(Ben, je pense que cela pourrait nous convenir.)

\section{Préambule}

- connecteur/ligateur

- point de vue

No (Ben)

- modus dissocié

mislim (je pense)

- cadre

nam ((a) nous)

- support lexical disjoint

/

\section{Rhème}

to bi ustrezalo. (cela pourrait convenir.)

Le préambule slovène est beaucoup plus condensé que le préambule français. Ce qui est typique, c'est que le ligateur ou le connecteur est le plus souvent exprimé par une particule modale, qui, à son tour, en même temps sert de lien et exprime l'attitude de l'énonciateur (Schlamberger-Brezar, 1996). La particule modale, comme p. ex. no,

7 On distingue le rapport de consensualité et de rupture, comme nous allons le voir par la suite. 
cité dans l'exemple (2) ci-dessus, couvre deux segments du préambule français, le connecteur/ligateur et le point de vue. Aussi le support lexical disjoint était très rare en discours analysés.

Ce qui est important pour notre recherche, c'est que les particules modales, qui prennent la position privilégiée au début du paragraphe, modifient tout le paragraphe et par conséquent agissent sur l'énonciation. Elles peuvent être apparentées à des adverbes énonciatifs. La position au début leur attribue le rôle préparatoire du thème (Hoye, 1997: 145). Du point de vue de l'intonation, elles sont, en position initale, habituellement marquées par une intonation montante et une pause, ce qui à l'écrit est noté, par approximation, par une virgule.

Si les particules modales apparaissent dans le préambule, ce qui est d'ailleurs souvent le cas en slovène parlé, elles ressemblent aux adverbes français qui expriment la modalité épistémique et agissent au niveau de l'énonciation. En plus, ce qui en slovène peut être exprimé par une seule particule à deux fonctions communicatives différentes, est en français souvent exprimé par le connecteur en combinaison avec un adverbe de modalité épistémique, comme p. ex. alors effectivement. Nous allons parler de cela dans la suite.

\section{Les marqueurs modaux et les connecteurs}

Le préambule se compose d'éléments qui, entre eux, ne sont pas en relation syntaxique, mais simplement juxtaposés. L'absence de marque syntaxique parmi les composants du préambule, d'après M. A. Morel (1998: 37) implique un ordre absolument fixe qui seul peut maintenir la différence de valeur entre les éléments.

Le préambule est en même temps le siège de la modalité épistémique, exprimant l'attitude de l'énonciateur envers ce qui est énoncé. Dans le modus dissocié est défini le garant de l'énonciateur pour la vérité et la certitude de l'information qui va suivre. La modalité épistémique est donc intégrée dans le thème (Hoye, 1997: 145), ce qui a été déjà souligné par M. A. K. Halliday (1985: 145), qui disait à propos des marqueurs modaux qu'ils sont habituellement inclus dans le thème:

"Si le locuteur fait inclure dans son message quelques éléments qui expriment son propre point de vue dans la matière, pour lui, ça va de soi de prendre son point de vue comme le point de départ." 8

Cette opinion ne diffère pas de la théorie de M.A. Morel et L. Danon-Boileau. La modalité déontique, à son tour, est intégrée dans le rhème.

Traditionnellement, les adverbes modaux et aussi les particules forment deux groupes selon la fonction qu'ils jouent dans l'énoncé: ils peuvent modifier soit le groupe de mots, soit la phrase, c'est-à-dire l'énoncé d'après les théories énonciatives. La modalité qui est repérée dans le préambule fonctionne comme la modalité énonciative.

8 Notre traduction, le texte anglais est le suivant: 'If the speaker includes within the message some elements that express his own angle of judgment on the matter, it is natural for him to make this his point of departure.' 
Dans le préambule se déroule aussi le double jeu de l'anticipation du coénonciateur et co-locuteur (Morel, Danon-Boileau, 1998: 9), c'est-à-dire des attentes et des objections que peut avoir celui à qui le discours s'adresse, c'est la coénonciation; mais aussi l'anticipation de la revendication du droit de chacun à la parole d'autre part, c'est la co-locution. Ces deux niveaux de représentation jouent un rôle essentiel dans la production du discours et sont codés dans chaque paragraphe.

Le schéma de la coénonciation a deux pôles dans lesquels sont distribués les connecteurs: la coénonciation et la rupture. Dans la coénonciation, les connecteurs donc et alors expriment la position consensuelle de l'énonciateur et du coénonciateur, tandis que le connecteur mais exprime la discordance. Dans la position de rupture, l'énonciateur ne prend pas en compte le coénonciateur; il s'agit donc du positionnement égocentré, qui est typique pour l'énonciation du connecteur et: ${ }^{9}$ ce qui va suivre est présenté comme inattendu.

Tous ces connecteurs qui commencent le paragraphe ont une fonction dans la coénonciation et jouent plutôt le rôle des marqueurs de la structuration discursive que le rôle de la connexion logique. A ces connecteurs sont ajoutés les marqueurs de la modalité épistémique, qui expriment soit le jugement, soit l'évidence. ${ }^{10}$ Le jugement est exprimé par les adverbes effectivement, sûrement, justement et des formes verbales je crois, je pense, et l'évidence par les adverbes évidemment et finalement.

\subsection{Les connecteurs et les marqueurs du jugement}

L'adverbe effectivement est un marqueur de jugement de l'énonciateur à propos de la valeur de vérité de la proposition énoncée. Cet adverbe implique un agent qui recentre l'énonciation sur sa propre position et exprime le jugement de la vérité de l'énonciation qui va suivre. Il apparaît dans les combinaisons suivantes: alors effectivement, mais effectivement, parce que effectivement. L'énonciateur lui-même est le garant de la vérité de l'énonciation, comme on peut voir dans l'exemple (3):

(3)

BM: Pour le reste, le problème que vous avez évoqué, qui est ... existe en effet c'est que nous, nous sommes fiables, nous faisons ce que nous avons dit, et que M. X dit qu'il n'a pas fait ce qu'il a fait. Alors effectivement ça ne simplifie pas les choses.

Dans l'exemple (3) le connecteur - marqueur de la structuration du discours alors implique la position consensuelle de l'énonciateur et du coénonciateur. A l'aide de l'adverbe effectivement, l'énonciateur recentre le discours sur sa propre énonciation et

9 Il faut distinguer le et conjonction, qui unit deux éléments de la même fonction syntaxique du et connecteur qui introduit le changement du sujet discursif ou la rupture avec ce qui a été dit auparavant.

10 D'après Palmer (1986), la modalité épistémique représente soit le jugement sur la certitude de l'énoncé, soit l'évidence que le locuteur a pour son énoncé. 
donne la garantie de la vérité de l'énonciation, qui ne fait pas partie du consensus avec le coénonciateur. En slovène, ce connecteur peut être traduit par la particule no avec l'intonation montante.

L'adverbe justement exprime le jugement de la précision du fait exprimé. Il apparaît comme alors justement et mais justement. Dans l'exemple (4), le connecteur mais inverse les points de vue. Il est mis en valeur par l'adverbe modal justement, qui réoriente la valeur argumentative de l'énoncé. En slovène, cette combinaison du connecteur et du marqueur peut être traduite par deux particules modales, no avec l'intonation tombante et $p a$, qui exprime l'opposition.

MC: Mais écoutez, les agriculteurs ne sont pas des jardiniers, ce sont des producteurs, des commerçants!

MHA: Bien sûr! Mais justement, ni le producteur ni le consommateur ne s'y retrouvent.

\subsection{Les connecteurs et les marqueurs d'évidence}

Le marqueur d'évidence évidemment apparaît dans alors évidemment (exemple (5)):

(5)

PL: C'est-à-dire?

MC: C'est-à-dire conclure un accord ou quoi?

BM: Il dit qu'il n'a pas conclu d'accord avec le Front National alors que ... à l'évidence, il en a conclu un. Alors évidemment ça ne simplifie pas les choses... pour lui, surtout pour lui.

L'adverbe évidemment est un adverbe-type de l'expression d'évidence visuelle. En slovène l'évidence est marquée par les adverbes očitno, jasno, et la particule modale seveda. ${ }^{11}$ L'acceptation de l'évidence dans l'exemple (5) est totale et l'évidence est placée dans le champ consensuel. Les énonciateurs n'ont pas besoin de discuter la vérité de l'énonciation, puisque c'est évident. L'évidence visuelle est le garant irréfutable pour la vérité dans notre civilisation, vu les expressions figées comme cela saute aux yeux, crève les yeux (en slovène to bode $v$ oči).

Dans notre corpus, nous n'avons pas trouvé la combinaison mais évidemment, qui, pourtant, est très fréquente en français. Les expressions de ce genre sont dangereuses pour le coénonciateur, parce qu'il s'agit d'un faux pas, d'une attaque argumentée jetée à la face du coénonciateur.

${ }^{11}$ La particule modale slovène seveda a ses racines dans le verbe vedeti - savoir - et exprime l'évidence cognitive. 
L'adverbe modal finalement exprime l'évidence qui est le résultat de la déduction: finalement $p$ veut dire que l'énonciateur a parcouru toutes les occurrences de $p$ et il n'a pas trouvé de valeur opposée, donc il peut finalement conclure $p$.

Dans l'exemple (6), la combinaison de connecteur donc, exprimant la déduction logique, est soulignée par finalement, qui, à son tour, lui aussi, apporte le résultat de la déduction: l'énoncé est irréfutable et consensuellement approuvé par l'énonciateur et le coénonciateur.

(6)

CD4: Oui, elle est écrasée par les convenances sociales d'ailleurs comme tous les autres, finalement, comme les autres personnages du roman... C'est pas seulement les femmes, les garçons aussi sont écrasés par des convenances sociales. Il y a un garçon qui s'enfuit sur la mer et qu'on ne revoit pratiquement jamais, qu'on ne revoit jamais. Il y a une fille qui se laisse mourir, il y a aussi un garçon qui se laisse mourir, toujours à cause des convenances sociales. Donc finalement Julia c'est une grande menteuse. Elle ment tout le temps, parce que sinon elle ne peut pas vivre.

Tous les adverbes mentionnés au-dessus expriment une forte modalité épistémique, soit comme jugements, soit comme évidentiels. Nous allons comparer leur rôle avec les particules modales de la langue slovène.

\subsection{Les particules modales slovènes}

En slovène, la coénonciation est gérée par les particules modales en position initiale et aussi par l'intonation: c'est le cas de la particule no, qui peut être traduite en français par oui, bon, ben, alors et qui peut, selon l'intonation que l'énonciateur va utiliser, exprimer soit la position consensuelle (exemple (8)) si l'intonation est montante, soit le désaccord (exemple (9)), si l'intonation tombe:

(8)

No, in na žalost...

Oui, et malheureusement...

No, saj, saj, to je v redu, da pač...

Ben, oui, oui, c'est bien que...

Dans l'exemple (10), les particules modales že že expriment la concession et sont suivies par le connecteur ampak (mais), exprimant le désaccord. Le mouvement discursif va de la concession à l'opposition.

A: Naša mreža res pokriva velik del celotnega tržišča.

B: Že že, ampak ko je Slovenija tak mali trg. 
A: Notre chaîne s'étend sur une grande partie du marché slovène.

B: Oui, mais la Slovénie en effet ne représente qu'un marché très restreint...

L'exemple (10) est intéressant du point de vue de la distribution de la particule modale et du connecteur : en français, comme nous venons de le voir, le connecteur précède l'adverbe modal. En slovène, le connecteur suit la particule modale.

\section{Conclusion}

Nous pouvons vérifier la première hypothèse : l'analyse en paragraphes oraux est applicable aussi à la langue slovène. Il est surprenant que ce genre d'analyse donne de meilleurs résultats pour le traitement des particules modales que toutes les autres théories (notamment celle de Toporišič, 1991) et mette en évidence les points communs du français et du slovène parlés. Le préambule slovène est plus condensé que le préambule français, mais il contient les mêmes éléments initiaux, le connecteur et le modus dissocié, comme on peut voir dans l'exemple (2) ci-dessus.

Le paragraphe slovène commence par une particule qui unit les fonctions des connecteurs discursifs et des marqueurs modaux. La théorie de Toporišič (1991) ou ces particules sont nommées "phrases rétrécies" ne rendait pas compte de leur valeur connective ou modale. Ces deux valeurs, à notre avis essentielles, sont mises en évidence par le modèle d'analyse de M. A. Morel et L. Danon-Boileau.

Dans le discours oral, au niveau de l'énonciation, les relations entre les paragraphes sont beaucoup plus souvent exprimées par les particules modales que par les conjonctions. C'est pourquoi le nom de "connecteur" ou même "ligateur" qui unit les deux catégories grammaticales sous une fonction linguistique, est beaucoup plus approprié pour la dénomination de ces liens que le terme "conjonction".

En français, c'est l'adverbe modal qui suit le connecteur. En slovène, le connecteur suit parfois la particule modale (p. ex. ̌̌e že, ampak) ou la particule même assure les deux fonctions. Sémantiquement, la fonction modale des particules est double : les particules sont premièrement modales et puis facteurs de liaison entre paragraphes. Le même effet peut être obtenu en français avec la combinaison du connecteur et de l'adverbe modal.

Ces conclusions sont importantes pour la traduction et pour l'apprentisage du slovène et du français. Le rôle des particules, défini dans ce cadre, apporte une solution pour la traduction des adverbes énonciatifs et encourage les apprenants du slovène à l'utilisation des particules dont la position peut être anticipée au début du paragraphe.

\section{Références}

ADAM, J. M. (1991) Eléments de linguistique textuelle. Bruxelles: Mardaga.

Berendonner, A.(1990) Pour une macro-syntaxe. Modèles linguistiques 21, p. 21-31.

DIK, S. C. (1989) The Theory of Functional Grammar. Dordrecht, Providence: Foris.

HALlidAY, M. A. K. (1985) Introduction to Functional Grammar. London: Edward Arnold.

HoYe, L. (1997) Adverbs and Modality in English. London, New York: Longman. 
HyberTIE, C. (1996) La conséquence en français. Paris: Ophrys.

LUSCHER, J.-M.(1994) Les marques de connexion: des guides pour l'interprétation. Dans Moeschler J. et al. Langage et pertinence. Nancy: PUN.

MORel, M. A. (1996) La concession en français. Paris: Ophrys.

MOREL, M. A. et al. (1997) Intonation, oral spontané (Comparaison de langues). Actes du CILIG. Paris 1997: CD Rom, Elsevier.

MORel, M. A., L. Danon-Bolleau (1998) Grammaire de l'intonation: exemple du français. Paris: Ophrys.

Palmer, R. (1986) Mood and Modality. Cambridge: Cambridge University Press.

Reboul, A., Moeschler, J. (1998) Pragmatique du discours. Paris: Armand Collin.

SPERBER, D., WILSON, D. (1986) Relevance: communication and cognition. Oxford: Basil Blackwell.

SCHIFFRIN, D. (1987) Discourse markers. 2e édition. Cambridge: Cambridge University Press.

SCHLAMBERGER BREZAR, M. (1996) Slovene negotiation conversation with stress on modality and argumentation. Abstracts of the $30^{\text {th }}$ International pragmatics conference. Antwerp: Ipra.

SCHLAMBerger BreZAR, M. (1998) Vloga povezovalcev v diskurzu. Dans Štrukelj, I. Jezik za danes in jutri. Zbornik referatov na II. kongresu Drustva za uporabno jezikoslovje. Ljubljana

SCHLAMBERGER BreZAR, M. (1999) Le rôle des topoï dans la négociation conversationelle. Linguistica XXXIX, p. 123-136.

TOPORıšıč, J. (1991) Slovenski členki in njihovi stavčni ustrezniki. Seminar slovenskega jezika, literature in kulture.

Povzetek

\section{POVEZOVALCI V POVEZAVI Z ZAZNAMOVALCI ZA EPISTEMIČNO MODALNOST: PRIMER FRANCOŠČINE IN SLOVENŠC̆INE}

Če si v raziskavah govorjenega diskurza pomagamo $\mathrm{z}$ analizo na enote kot so stavek, poved, pa tudi govorno dejanje, ne dobimo zadovoljivega odgovora na vprašanje, katere enote med seboj vežejo povezovalci. V okviru analize, ki jo v francoskem jezikoslovju uvajata M. A. Morel in L. DanonBoileau, je podana enota govorjenega diskurza, imenovana odstavek, ki je določena intonacijsko, diskurzivno in oblikoslovno-skladenjsko. Ta enota se deli na wvodni del, ki ni skladenjsko strukturiran, remo, kjer je ohranjena skladenjska struktura, in postremo. V uvodnem delu odstavka so izpostavljeni povezovalci in zaznamovalci za epistemično modalnost. Če enoto apliciramo na slovenski govorjeni diskurz, v uvodnem delu pridejo do izraza slovenski členki, ki hkrati delujejo kot povezovalci in zaznamovalci za epistemično modalnost. $V$ francoskem jeziku se povezave te vrste vzpostavljajo s povezovalci $\mathrm{v}$ kombinaciji s prislovi, ki izražajo epistemično modalnost, torej sodbe (kot na primer alors effectivement, mais justement) ali dokaznost (alors évidemment). Paralele te vrste lahko služijo za boljše slovnične opise delovanja povezovalcev in zaznamovalcev za modalnost ter členkov in so dragocene tako za prevajanje kot tudi za poučevanje slovenščine kot tujega jezika. 